\title{
Man sollte darüber reden
}

\section{Aktiv gegen das stille Volksleiden Inkontinenz}

„Darüber reden statt darunter leiden!“ ist das Motto der Medizinischen Kontinenzgesellschaft, das auch für die Welt Kontinenz Woche, vom 18. bis 24. Juni 2012 im Mittelpunkt stand. Ausgerufen von der International Continence Society (ICS) soll die Initiative weltweit die Sprachlosigkeit, die immer noch um das tabubehaftete Thema Inkontinenz herrscht, aufbrechen und Betroffene motivieren Hilfe in Anspruch zu nehmen. Die MKÖ bot Information sowie kostenlose persönliche und telefonische Beratung. Die 22. Jahrestagung der MKÖ Mitte Oktober wird den Fachleuten den aktuellen Stand des Wissens und Diskussionen darüber ermöglichen.

Inkontinenz ist weit verbreitet. Etwa eine Million Österreicher haben Probleme mit der Blase oder dem Darm. Frauen sind weitaus häufiger betroffen und mit dem Alter nimmt die Wahrscheinlichkeit, die Blase oder den Darm nicht mehr ausreichend unter Kontrolle zu haben, naturgemäß zu. Doch auch in jungen Jahren - z. B. nach einer Schwangerschaft oder einer Prostata-Operation - können Probleme auftreten. „Die Hälfte der Erkrankten ist unter 60 Jahre alt", stellt Dr. Mons Fischer, Präsident der Medizinischen Kontinenzgesellschaft Österreich (MKÖ), fest. Sogar Kinder sind betroffen: „Bettnässen und kindliche Harninkontinenz zählen nach Allergien zu den häufigsten chronischen Erkrankungen im Kindesalter."

Bei Inkontinenz gibt es viele unterschiedliche Formen und Ursachen, die aber alle eines gemein haben: Sie bringen einen enormen Leidensdruck mit sich. Es kommt zur Beeinträchtigung des Alltags und des sozialen Lebens: „Angst davor, dass die Umgebung etwas sieht oder riecht, Ausrichtung des täglichen Lebens um die Nähe zu Toiletten, finanzielle Belastungen durch Hilfsmittel und Wäschepflege sind ein kleiner Auszug, wie es Betroffenen geht", berichtet Fischer. Dennoch: Aus Scham und weil zu wenig Wissen über die gute Behandelbarkeit von Blasen- und Darmschwäche vorhanden ist, geht nur ein Bruchteil zum Arzt und holt sich medizinische Hilfe. Viele meiden die Öffentlichkeit und verlieren zunehmend ihr Selbstwertgefühl. Auch Angehörige sind durch die Situation sehr belastet und häufig ebenso rat- und hilflos. Fischer: „Blasen- und ganz besonders Darmschwäche sind nach wie vor ein Tabuthema in unserer sonst so aufgeklärten Gesellschaft.“

\section{Inkontinenz ist behandelbar}

Gegen Blasen- und Darmschwäche kann man in den meisten Fällen etwas tun! Je

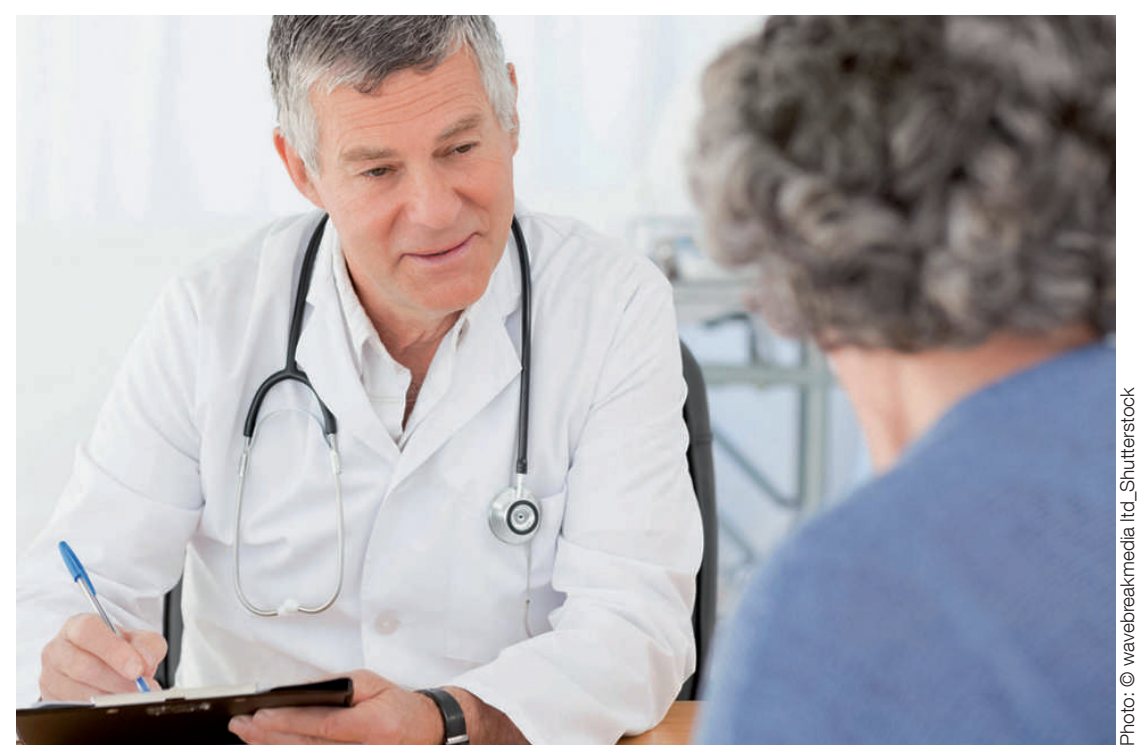

Vertrauen zwischen Patient und Arzt muss aufgebaut werden. nach Ursache gibt es heute eine Reihe von medikamentösen, physikalischen, operativen und alternativen Behandlungsmöglichkeiten. „Je früher mit der Therapie begonnen wird, desto besser sind die Heilungschancen“, appelliert Fischer. „Besonders wichtig vor Beginn der Behandlung ist die Erkennung der Inkontinenzform, damit sie zielgerichtet erfolgen kann. Über die MKÖ können die richtigen Anlaufstellen in allen Bundesländern im ambulanten und niedergelassenen Bereich abgerufen werden." Mit richtigem und rechtzeitigem Beckenboden- und Blasentraining können verschiedene Inkontinenzformen in vielen Fällen wesentlich gemildert bzw. überhaupt vorgebeugt werden.

\section{Neue Therapien bringen neue Zuversicht}

„Auf dem Gebiet der Blasen- und Darmfunktionsstörungen wurde in den vergangenen Jahren viel Forschungsarbeit geleistet. Neue Entwicklungen in der Inkontinenzbehandlung und -versorgung ermöglichen Betroffenen wieder ein aktives Leben", so Fischer und nennt ein paar Beispiele: „Ist richtig durchgeführtes Beckenbodentraining bei der Belastungsinkontinenz nicht ausreichend erfolgreich, können nun neu entwickelte minimal invasive Operationsmethoden zum Einsatz kommen, die kaum belasten und in jedem Alter durchgeführt werden können." Für die Dranginkontinenz wurden besser verträgliche Medikamente entwickelt: „Es gibt wirksame Substanzen, die mittels Katheter direkt in die Blase eingebracht werden. Auch Botox, das in die Blasenwand gespritzt wird, kann jetzt Linderung bringen“, so der Urologe. „Eine Elektrostimulation über die Haut oder direkt am Nerv über ein implantiertes Stimulationsset (Neuromodulation) kann die massiven Harndrangbeschwerden beherrschen."

Anlässlich der diesjährigen Aktionswoche startete die MKÖ auch eine facebook fanpage: www.facebook.com/kontinenz. „Diese Seite wird von einer Kontinenzschwester betreut und ist ein Informations- und Austausch-Portal für alle Interessierten, das einen weiteren wichtigen Beitrag zur Enttabuisierung leisten kann", stellt Fischer fest.

Quelle: Presseinformation Medizinische Kontinenzgesellschaft Österreich 